International Journal of Engineering \& Technology, 7 (2.7) (2018) 51-53
International Journal of Engineering \& Technology
SPC
Website: $w$ ww.sciencepubco.com/index.php/IJET
Research Paper

\title{
Website evaluation using opinion mining
}

\author{
T.V.R. Sai ${ }^{1}$, Sk. Haaris ${ }^{2}$, S. Sridevi ${ }^{3}$ \\ ${ }^{1}$ Department of electronics and computer engineering KLEF Deemed to be University \\ ${ }^{2}$ Department of electronics and computer engineering KLEF Deemed to be University \\ ${ }^{3}$ Asst Professor Department of electronics and computer engineering KLEF Deemed to be University \\ *Email: saitvr8@gmail.com
}

\begin{abstract}
In this project we used opinion mining methods to evaluate various websites present on the internet. We also analyzed the approaches, tools, and dataset used by Scholars with their accuracy and we used this technology for evaluation of a website. Opinion mining is used in various scenarios around the world. But it is hardly used in websites evaluation which we are implementing with this project, as now a day's, websites we regularly use are spamming with advertisements and unusable content. This paper proposed a frame work of evaluating a website using the user feedback on the website collected on our website. That collected feedback data is processed using a data mining software that is rapid miner
\end{abstract}

Keywords: Opinion Mining, Data Mining, Sentiment Analysis, Text Processing, Website Development.

\section{Introduction}

Generally, people and corporations are invariably fascinated by other's conclusion like on the off chance that some individual needs to get a spic and span item, at that point to start with, he/she tries to get a handle on the audits i.e., what individuals have certainty the stock and upheld those surveys, he/she takes the decision. Similarly, corporations conjointly excavate deep for shopper reviews. But such giant and big info on the net is of no use for a corporation till the accumulated content being not extracted and analyzed properly. [1]However, to come up with profits or to learn from these opinions and experiences, Opinion mining is one in all the approaching fields of the reviewers a few specific product, topic or services. It refers to a group of techniques that deals with information regarding opinions and tries to realize valuable info from them. There are 2 main classes of matter information: facts and opinions. Facts are objective expressions regarding events, entities and their properties.

\section{Rapid Miner}

Opinion mining, because the name implies, is that the mining (extracting) of opinions regarding the merchandise, event, services, etc. announce by variant folks on the net.[2] Thanks to the large enlargement of the net, folks are inspired to contribute themselves with blogs, social networking sites, etc. an outsized volume of knowledge is created thanks to these platforms that require being well-mined (extracting helpful patterns) for analysis and higher cognitive process. Opinion-mining systems analyses regarding the reviews like that half is opinion conveying, its various selection of administrators, Rapid Miner Likewise incorporates the information mining library from the WEKA Toolkit.

\section{Procedure}

\subsection{Rapid miner setup for opinion mining:}

Rapid miner is an open source information mining and learning disclosure instrument written in Java, joining most understood digging calculations for arrangement, grouping, and relapse; it additionally contains modules for specific assignments, for example, content mining and examination of gushed information. Fast Miner is a GUI based device, however, mining assignments can likewise be scripted for cluster mode preparing.[3] Notwithstanding 3.2. Title and author details. The extremity informational index is an arrangement of surveys of a site gathered on our site, which was named in view of creator input: positive or negative. There are 100 named records for each class, and the information is displayed in the plain content organization. [4]This informational collection has been utilized to investigate the execution of supposition mining strategies.

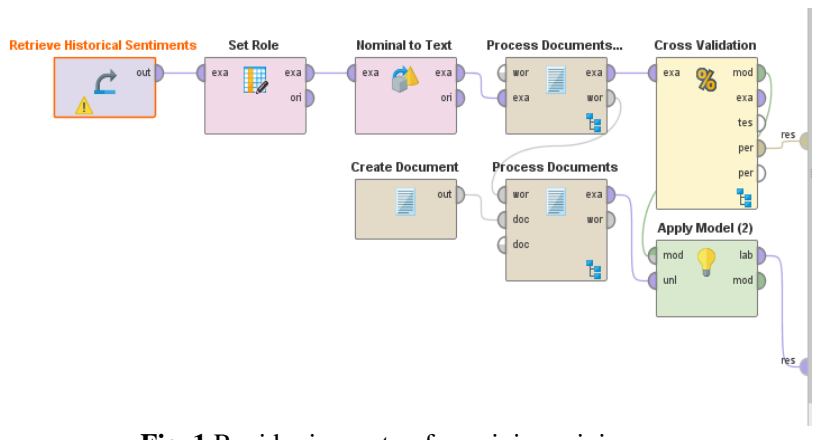

Fig. 1 Rapid miner setup for opinion mining 


\subsection{Retrieve Historic Sentiment:}

The Retrieve Operator loads a Rapid Miner Object into the Process. This Object is often an Example Set but it can also be a Collection or a Model Retrieving data this way also provides the Meta data of the Rapid Miner Object.

\begin{tabular}{|l|l|l|}
\hline Row No. & Sentiment & Text \\
\hline 1 & negative & Bad \\
\hline 2 & positive & good \\
\hline 3 & negative & useless \\
\hline 5 & positive & awesome \\
\hline 6 & positive & useful \\
\hline 7 & positive & helpful \\
\hline 8 & positive & best \\
\hline 10 & negative & ads \\
\hline
\end{tabular}

Fig 2. List of words that are classified as positive or negative

\subsection{Set Role}

The part of an Attribute characterizes how different Operators switch this Attribute. The default role is regular, other roles are classified as special. An Example Set can have many singular Attributes, but each singular role can only appear once. If a singular role is assigned to more than one Attribute, all roles will be changed to regular except for the last Attribute. The different types of roles are clarified below in the parameter section.

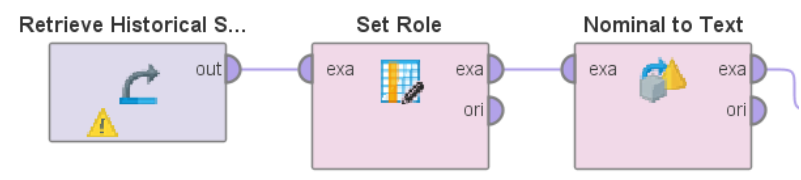

Fig 3. Attributes from Retrieve Historic Sentiment to Set Role

\subsection{Nominal to Text}

The Nominal to Text administrator changes over every single ostensible credit to string traits. Every ostensible esteem is just utilized as a string estimation of the new quality. On the off chance that the esteem is absent in the ostensible quality, the new esteem will likewise be missing.

\subsection{Process Documents}

The process documents consist 3 stages as shown below

\section{Tokenize}

\section{Transform cases}

3. Filter Stop words

Process Documents from Data

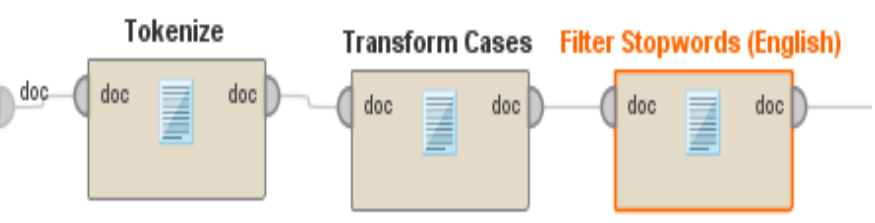

Fig 4. Process document blocks

\subsubsection{Tokenize}

This operator parts the substance of a report into a no.of tokens. There are a couple of choices how to show the part centers. Perhaps you may use all non-letter character, what is the default settings. This will achieve tokens including one single word, what's the most suitable decision before finally assembling the word vector Or if you will build windows of tokens or a comment impact, you will apparently part whole sentences, this is possible by setting the split mode to demonstrate character and enter each part character. The third decision permits you to portray typical enunciations and is the most versatile for particularly exceptional cases. Each non-letter character is used as the separator. Along these lines, each word in the substance is addressed by a single token.

\subsubsection{Transform cases}

This operator changes all characters in a file to either cut down the case or promoted, independently.

\subsubsection{Filter Stopwords}

This operator channels English stopwords from a report by ousting every token which squares with a stopword from the natural stopword list. If it's not too much trouble take note of that, for this administrator to work appropriately, every token ought to speak to a solitary English word as it were. To acquire an archive with every token speaking to a solitary word, you may tokenize a report by applying the Tokenize administrator heretofore.

\section{Validation blocks}

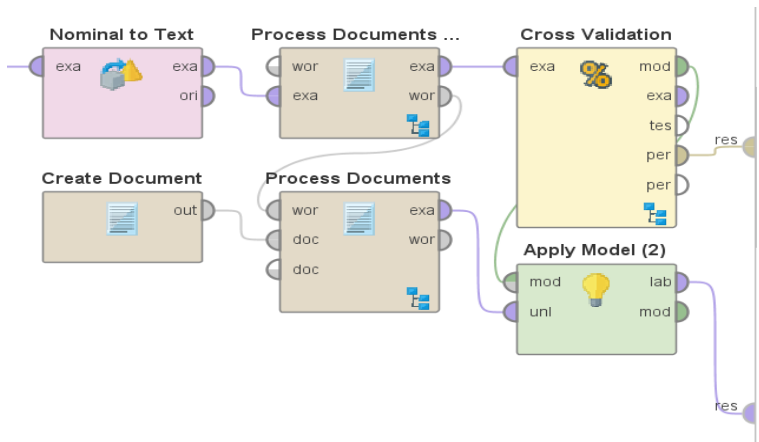

Fig 5. Validation blocks

\subsection{Cross-validation}

It is, for the most part, used to estimation how decisively a model (learned by a particular learning Operator) will perform eventually. 
The Cross Validation Operator is a settled Operator. It has two subprocesses: a Training subprocess and a Testing subprocess. The Training subprocess is used for setting up a model. The readied demonstrate is then associated with the Testing subprocess. The execution of the model is evaluated in the midst of the Testing stage.

The data Example Set is distributed into k subsets of equal size. Of the $\mathrm{k}$ subsets, a singular subset is held as the test instructive accumulation (i.e. commitment of the Testing sub process). [5]Whatever is left of the $\mathrm{k}-1$ subsets are used as getting ready educational gathering (i.e. commitment of the Training sub process). The crossendorsement process is then repeated $\mathrm{k}$ times, with each of the $\mathrm{k}$ subsets used accurately once as the test data. The $\mathrm{k}$ comes about because [6] The assessment of the execution of a model on autonomous test sets yields a decent estimation of the execution on concealed informational indexes. It additionally appears if 'overfitting' happens. This implies the model speaks to the testing information extremely well, however it doesn't sum up well for new information. Hence, the execution can be much more awful on test information.

\section{Working}

step1: Initially data is collected from the use that is the comment as follows

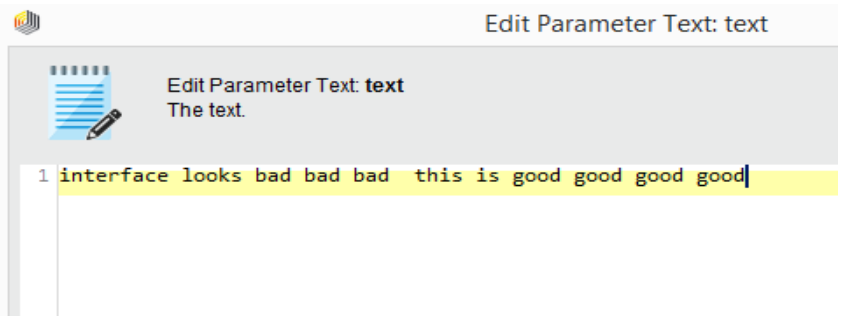

Fig 6. Collected data from user

Step2: The collected data that is the comment from the user is processed into the stage as described in the process document.

Step3: The above-analyzed data is compared with the historic data and the positive and negative data points are assigned accordingly in the rapid manner tool this is termed to be opinion mining done in this tool used.

\section{PerformanceVector}

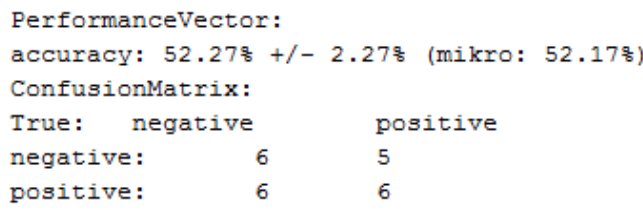

Fig 7. Performance vector

\section{Results}

The collected reviews of a particular website for evaluation are loaded into the process document and the given data is processed withe given historic sentiment data and the out of the process is a categorization of the given review into the positive review or a negative review based on the words in the sentences. The output of the processed data in the form of performance vector is given below. project2 $(2$ results. Process results)

Completed: Jan 25, 2018 2:06:38 PM (execution time: $0 \mathrm{~s}$ )

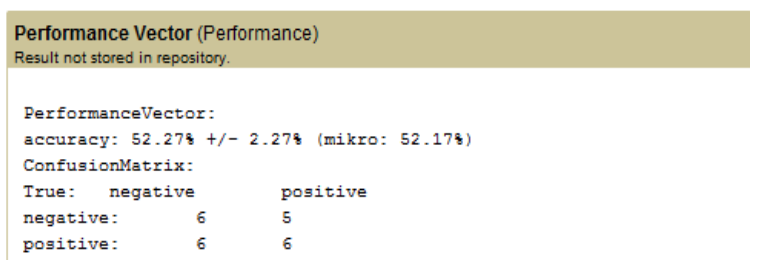

Fig 8. Performance vector (performance)

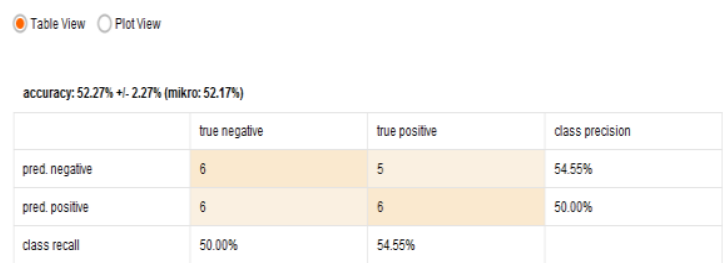

Fig 9. Accuracy of the result

\section{Conclusion}

we have observed that "website evaluation using opinion mining" has given great results in extraction of information from the huge date that in sense is the comment from the user and analyzing it to be overall positive or negative .which is done by the rapid miner tool and results are acquired accordingly shown above.

\section{Reference}

[1] Ion SMEUREANU, Cristian BUCUR, Applying Supervised Opinion Mining Techniques on Online

[2] "Opinion Mining and Sentiment Analysis in Information Retrieval Vol. 2, Nos. 1-2 (2008), Bo Pang and Lillian Lee.

[3] "Affect intensity analysis of dark web forums," in Proceedings of Intelligence and Security Informatics (ISI), pp. 282-288, 2007, Abbasi.

[4] Mining the Peanut Gallery: Opinion Extraction and Semantic Classi_cation of Product Reviews." Proceedings of the 12th International Conference on World Wide.

[5] B. Pang \& L. Lee, ISeeing stars: Exploiting class relationships for sentiment categorization with respect to rating scales." Proceedings of the Association for Computational Linguistics (ACL), pp.

[6] Towards Enhanced Opinion Classification using NLP Techniques, IJCNLP 2011, pages 101-107, Chiang Mai, Thailand, November 13,2011

[7] Gautami Tripathi and Naganna S, "FEATURE SELECTION AND CLASSIFICATION APPROACH FOR SENTIMENT ANALYSIS", Machine Learning and Applications: An International Journal (MLAIJ) Vol.2, No.2, June 2015

[8] LiZhen Liu, Zhixin Lv, -Opinion mining based on feature-level, Image and Signal Processing (CISP)\|, 2012.

[9] Bing Liu, 2012, Sentiment analysis and opinion mining, Morgan and Claypool publishers.

[10] Das, Sanjiv and Mike Chen, 2007, Yahoo! for Amazon: Sentiment extraction from small talk on the web, Management Science, 53(9): 1375-1388.

[11] HaseenaRahmath P, "Opinion Mining and Sentiment Analysis Challenges and Applications", International Journal of Application or Innovation in Engineering \& Management (IJAIEM), Volume 3, Issue 5, May 2014

[12] Ananta Arora et al, "Opinion Mining: An Overview", International Journal of Advanced Research in Computer and Communication Engineering Vol. 4, Issue 11, November 2015.

[13] Solanki Yogesh Ganeshbhai, Bhumika K. Shah, "Feature Based Opinion Mining: A Survey", IEEE International Advance Computing Conference (IACC), 2015. 